\title{
ESTRATEGIAS METODOLÓGICAS PARA EL ENRIQUECIMIENTO LÉXICO EN ESTUDIANTES DE TERCER CICLO DE LA EDUCACIÓN GENERAL BÁSICA
}

\author{
Gisela Vega Castro \\ Profesora del Liceo Enrique Güier Sáenz
}

Recibido 11-VIII-2005 • Aceptado 13-IX-2005

\begin{abstract}
Resumen: El presente artículo ofrece los resultados de un estudio de tesis, cuyos objetivos principales se basan en poner en práctica diversos métodos didácticos para la enseñanza del léxico en el nivel de octavo año de la Educación General Básica, con el fin de determinar en qué medida estos son efectivos o no para enriquecer el léxico en los estudiantes.
\end{abstract}

Palabras clave: Léxico, lexema, disponibilidad léxica, competencia léxica, campos léxicos, aprendizaje significativo.

Abstract: This essay contains the results obtained while on a thesis-related paper. To this end, the objectives of the thesis were focused on the effectiveness of diverse didactic methods in terms of the acquisition of lexical terms among eighth graders.

Key words: Vocabulary, lexeme, lexical availability, lexical competence, lexical fields, lexical terms, meaningful learning.

\section{Introducción}

La competencia léxica que posee toda persona es un instrumento valioso que le permite desenvolverse en las diversas situaciones de la vida, tanto en el plano académico como familiar y, de esta manera, poder expresar de forma clara sus ideas, pensamientos, sentimientos y opiniones, todo con el propósito de entablar relaciones comunicativas con las demás personas que giran en torno a su ambiente y participar como miembro de una sociedad.

De acuerdo con observaciones que he podido realizar en mi labor docente y según diversos especialistas en el tema de educación, los estudiantes de secundaria poseen un vocabulario limitado, con el cual expresan sus ideas. Así como lo plantea Marta Rojas (1994) en su artículo de revista titulado Caracterización de los procesos de enseñanza-aprendizaje a partir del análisis de la interacción verbal en el aula costarricense, se debe fomentar el uso de un léxico amplio en el plano oral como escrito, tanto para la expresión y la recepción de mensajes.

"La concepción del idioma como instrumento de comunicación, base orientadora de la enseñanza del Español en nuestro país, enfatiza la importancia de desarrollar en el individuo, de igual manera, la mejor expresión y la más adecuada recepción." (Rojas, 1994:130). 
Si los individuos no tienen la posibilidad de ampliar su vocabulario es probable que no puedan alcanzar un domino de los registros escritos formales y con ello se les dificultarán las vías para expresarse de forma escrita y oral.

La propiciación del enriquecimiento léxico de los estudiantes debe hacerse bajo el directriz de técnicas que enseñen un vocabulario útil, dentro del contexto social y cultural en el que se desenvuelvan los alumnos, para que de esta manera el conocimiento de la lengua permita proporcionar una enseñanza de nuestra realidad social y lingüística, “...la enseñanza del léxico no debe aparecer descontextualizada. No se debe promover la noción de lengua ideal fuera de contexto." (Araya, 2000:88).

Dentro de los contenidos de los programas de estudio de la educación formal, no sólo de Español, sino también de Estudios Sociales, Matemáticas, Ciencias y el resto de las asignaturas, se debe prestar especial atención al desarrollo del léxico, como una manera de asegurar que los estudiantes puedan expresarse correctamente y que el enriquecimiento del vocabulario en los alumnos sea un arma que empleen y practiquen en las distintas circunstancias de su desempeño, tanto académico como personal.

Por lo anterior, la investigación de la que se presentan algunos resultados en estas páginas, pretendió mejorar el nivel de desempeño léxico, mediante actividades que les permitan a los jóvenes de Tercer Ciclo de la Educación General Básica adquirir un mayor dominio de la expresión escrita y oral.

\section{Marco teórico}

\section{Léxico}

Según el Diccionario de la Real Academia Española (DRAE), se puede definir el término léxico como "todo lo perteneciente o relativo al vocabulario de un idioma o región. El vocabulario es un conjunto de palabras en un idioma o términos que pertenecen al uso de una región, actividad o campo determinado."

Desde el punto de vista lingüístico, el léxico le permite, a los seres humanos, comunicar sus experiencias de vida y sus percepciones con respecto a los objetos que los rodean, hablar de sus emociones, sus conocimientos, comidas y bebidas, costumbres, trabajos, juegos y sus interacciones sociales; es decir, todo el vocabulario que las personas son capaces de manejar con el fin de expresarse, tanto de manera oral como por escrito, dentro de la sociedad y que es aprendido en el ámbito educativo, familiar y social.

De acuerdo con Sánchez (1993), en su artículo Campos semánticos y disponibilidad léxica en preescolares, el éxito que se tenga en la enseñanza del vocabulario está determinado por el proceso de adquisición del lenguaje oral.

“...la labor educativa debe fundamentarse en estudios de lingüística aplicada a la enseñanza de la lengua, que señalen las pautas por seguir en el desarrollo de las destrezas lingüísticas. Asimismo, es primordial conocer cuál es la competencia comunicativa del niño -vocabulario, sintaxis, discurso-y sobre la base de tales datos planificar la enseñanza de la lengua." (Sánchez, 1993:15).

El léxico es, además, un conglomerado de vocabularios, variedades y propiedades que utilizan los hablantes para comunicarse en diversas situaciones. Por tal motivo, es necesario que la incorporación del léxico sea un proceso organizado, de manera que se pueda avanzar en grados de conocimiento con la incorporación de términos y se refuerce el vocabulario ya existente en la estructura mental de las personas.

\section{Lexema}

Un lexema es cada una de las palabras que forman parte del caudal léxico o vocabulario concreto que el hablante utiliza 
en una situación comunicativa particular, es decir todo el vocabulario básico y el vocabulario disponible en una lengua.

La semántica es la disciplina encargada del estudio del contenido de las formas gramaticales de los lexemas, entendiendo estos, según Horst Geckeler (1976) en su texto Semántica estructural y teoría del campo léxico como "los miembros de un campo léxico" (Geckeler. 1976:297), es decir, las unidades léxicas que interactúan en un campo de significación o que comparten ciertos rasgos distintivos. Algunas de esas formas gramaticales de los lexemas son las siguientes: plural, singular, indicativo, presente, masculino, femenino, entre otras. Es decir, estudia las funciones de las palabras y no sólo su forma de expresión, por lo tanto, abarca el contenido de estas.

Cada una de las palabras o lexemas tiene un significado determinado y cualquier alteración en dicha palabra acarreará un cambio en el sentido o significado de esta. Por ejemplo: perro, significa, según la definición del diccionario, "mamífero doméstico de la familia cánidos"; si se hiciera una alteración en esta palabra, por dar un ejemplo, eliminarle una "r", dicho término se convertiría (según la definición del diccionario) en pero y por consiguiente su significado variaría de la siguiente manera: "conjunción adversativa con que a un concepto se contrapone otro diverso o ampliativo del anterior".

\section{Disponibilidad léxica}

Por disponibilidad léxica se entiende el caudal léxico o vocabulario que el hablante utiliza en un contexto comunicativo dado. En cada situación en la que se desenvuelvan los individuos, las palabras que acuden primero a la memoria son aquellas que tienen relación con el contexto en que se encuentre la persona y por lo tanto, son más disponibles en relación con la situación comunicativa dada, que aquellas palabras que no aparecen inmediatamente, de ahí, que el índice de disponibilidad sea una medida que una la frecuencia y el orden de aparición de dichas palabras.

La competencia léxica no sólo incluye la cantidad de conocimientos que una persona tenga del vocabulario, ya sea de su lengua materna o de un idioma extranjero, sino también las características y funciones que esas unidades léxicas puedan tener en todas las dimensiones del lenguaje: lingüística, discursiva, pragmática y referencial.

La frecuencia con que se utilicen los términos varía por el género o tema que se esté tratando, es decir, tendrá menor disponibilidad aquel léxico de palabras especializadas. Por ejemplo: “...algunos hablantes del nivel popular integrados en ciertas áreas de actividad, pescadores, agricultores, ganaderos, etc., manejarían un vocabulario más rico que los propios hablantes cultos y hasta que algunos escritores clásicos.” (Ortega, 1996:24-25).

\section{Competencia léxica}

La competencia léxica se concibe como el dominio de todas las características y funciones de las unidades léxicas, género, número, modos, tiempos, entre otros elementos; por ello, es necesario conocer las palabras y la situación en la que éstas pueden utilizarse, tomando en cuenta que, al ser el lenguaje un objeto de constante cambio, la disponibilidad léxica se renueva persistentemente, de acuerdo con las necesidades y conforme se utilice el vocabulario en las diversas situaciones sociales en las que el ser humano tiene que desenvolverse cada día: "...la disponibilidad señala el vocabulario concreto que el hablante podría utilizar en un contexto comunicativo dado.” (Ortega. 1996:23).

\section{Campos léxicos}

Desde el punto de vista semántico, la estructura léxica de una lengua o de su 
vocabulario, se entiende como una red de sentido, en la que cada palabra entabla una relación con el resto de términos dentro del campo semántico, ya sea por poseer rasgos distintivos semejantes o diferentes. Cada una de estas relaciones se anuda por medio de otro lexema o palabra, es decir, se interrelaciona con un nivel superior.

Desde el punto de vista estructural, un campo léxico es considerado como la "repartición de un contenido léxico continuo entre diferentes unidades dadas en la lengua como palabras y que se oponen de manera inmediata unas a otras, por medio de rasgos distintivos mínimos." (Coseriu, 1977:146).

Un campo léxico puede estar incluido dentro de otro campo léxico superior, precisamente por la capacidad que poseen las palabras de relacionarse dentro de un mismo campo y, por consiguiente, con diversos campos léxicos.

\section{Estrategias didácticas para el mejoramiento léxico}

Como paso fundamental para la aplicación de estrategias didácticas que promuevan o mejoren la enseñanza de una lengua se requiere la utilización de una teoría pedagógica adecuada a la situación de enseñanza-aprendizaje de los estudiantes, fundamentalmente que sea práctica, participativa y creativa, con el propósito de que los alumnos identifiquen las variedades lingüísticas existentes y puedan comentarlas y analizarlas.

De acuerdo con Garrido Moraga (1991), citado por Marielos Murillo (1999) en su artículo La enseñanza del vocabulario en la Educación General Básica: propuesta metodológica, la utilización del diccionario es el principal medio de enriquecimiento del vocabulario, pues su uso acarrea características de prestigio, seguridad y cohesión, ya que se supone, dicho instrumento es el depositario de todo el conocimiento léxico de un idioma o lengua.
Por otra parte, se encuentra el estudio del vocabulario mediante el análisis de rasgos semánticos. Este, con base en los postulados de Pittelman y otros (1991), citados por Marielos Murillo (1999), consiste en las interconexiones emocionales o semánticas que los estudiantes puedan entablar entre las palabras nuevas y el léxico ya conocido, es decir, llevar a cabo un aprendizaje significativo y entablar puentes cognitivos entre el conocimiento previo y el nuevo aprendizaje.

De acuerdo con el artículo, $A l$ principio ya existía la palabra. Caminos autónomos e individuales hacia la elaboración el aprendizaje del vocabulario, Vences (1996) plantea una serie de estrategias para el aprendizaje de vocabulario, entre estas se pueden citar las siguientes:

1. Deducir palabras: leer encabezados periodísticos o titulares para inducir el significado de ciertas palabras que los estudiantes desconocen, como una forma de ampliar el vocabulario con base en "internacionalismos".

2. Crear palabras: por medio de la analogía y la derivación, los estudiantes pueden formar nuevos términos.

3. Reflexionar sobre el significado de las palabras: analizar el significado $\mathrm{u}$ origen de ciertas palabras y relacionarlas con términos parecidos como: casarse - casa.

4. Relacionar palabras: es la búsqueda de campos semánticos en los que se puedan incluir antónimos o sinónimos y formar cadenas de palabras. Posteriormente, dichos términos serán utilizados en un contexto (frases u oraciones), con el propósito de que el vocabulario sea memorizado de una manera más práctica, por medio de la conversación o producción lingüística. 


\section{Aprendizaje significativo}

El aprendizaje significativo se refiere a la adquisición de nuevos significados de una manera potencial, es decir, que el material de aprendizaje se relacione de una forma no arbitraria y sustancial con el conocimiento previo que posea la persona o con algún aspecto relevante que el estudiante tenga en su estructura cognoscitiva.

Para Joseph D. Novak (1997), en su texto Teoría y práctica de la educación, es importante tomar en cuenta los conocimientos que el alumno ya tiene y a partir de ahí enseñar en una forma consecutiva para que así pueda darse un aprendizaje realmente significativo, de manera que los estudiantes relacionen los conocimientos que posean. Sin embargo, estipula que en el caso de no existir ningún tipo de conocimiento acerca de un tema determinado, el aprendizaje debe llevarse a cabo en una forma memorística, pues los alumnos no tendrían ningún referente conceptual con el cual crear un puente cognoscitivo para establecer semejanzas o diferencias con el saber aprendido.

"Se da un aprendizaje significativo, cuando la información nueva se pone en relación con conceptos ya existentes en la mente del que aprende (conceptos inclusivos o inclusores, en términos de Ausubel)." (Novak. 1977:25).

Dentro de los tipos de aprendizaje significativo que se han puesto en práctica, es el aprendizaje significativo por recepción el que más atención ha recibido por parte de diversos autores.

"El aprendizaje significativo por recepción es importante en la educación porque es el mecanismo humano por excelencia que se utiliza para adquirir y almacenar la vasta cantidad de ideas e información representada por cualquier campo del conocimiento." (Ausubel. 1989:47).

Dentro de este uso se pueden encontrar tres tipos distintos: el aprendizaje por representaciones, el aprendizaje por definiciones conceptuales o teóricas y el aprendizaje por proposiciones, que se concibe como la presentación de una circunstancia para que los estudiantes puedan encontrar soluciones a problemas que les plantea el docente.

a. El aprendizaje por representaciones: aprender el significado de símbolos o palabras. Se lleva a cabo mediante el aprendizaje de términos aislados, tomando en cuenta el significado al cual se puede referir el concepto.

b. El aprendizaje por conceptos: los conceptos también son representados por imágenes aisladas, para dar una mayor claridad visual de los términos y que estos puedan ser más comprensibles para los estudiantes.

c. El aprendizaje por proposiciones: se trata de captar el significado en ideas expresadas en forma de propuestas, las cuales deben poner de manifiesto ideas diferentes a sus equivalentes en representaciones concretas.

La meta que persigue el aprendizaje significativo es poder entablar relaciones entre la información que posean los alumnos y los datos nuevos que cada día van adquiriendo en cualquier campo del saber: ciencias, español, matemática, con el fin de que pongan en práctica esos conocimientos en sus quehaceres diarios.

\section{Procedimientos metodológicos}

\section{Informantes}

Cincuenta y tres estudiantes de octavo año en el colegio Saint Anthony High School, ubicado en Tibás, San José.

\section{Recolección de la información}

La base de la presente investigación son los diseños cuasi-experimentales, en los cuales, los factores que afectan el 
experimento se controlan de una forma no tan rigurosa y el investigador, aunque diseñe un experimento, no puede manipular todas las variables que entran en juego.

El grupo experimental es no autoseleccionado, sino escogido al azar, al igual que el grupo control. Al primero se le aplica una pre-prueba, para medir el conocimiento que los estudiantes tienen con respecto a un vocabulario determinado. Posteriormente, debía ser aplicada una pos-prueba, con el fin de comprobar la funcionalidad de las estrategias utilizadas en la enseñanza del léxico.

El corpus elegido para la puesta en práctica de métodos didácticos que enriquezcan el léxico en los estudiantes de secundaria fue tomado de dos de los cuentos que forman parte del plan de estudios de octavo año en la Educación General Básica: "El hijo" de Horacio Quiroga y "El difunto José” de Joaquín García Monge.

\section{Las pruebas}

Para medir el uso que los estudiantes hicieron de las ochenta palabras seleccionadas de los cuentos "El hijo" y "El difunto José" se confeccionó una pre-prueba que consiste en dos partes, la primera es una redacción que los alumnos debían escribir basándose en treinta y cinco palabras del corpus total de los textos. Estas palabras se seleccionaron para la redacción, pues la profesora investigadora consideró que podían pertenecer a campos semánticos similares y con ello se facilitaría al estudiante poder utilizarlas en un contexto escrito que se centrara en un tema determinado.

La segunda parte de la prueba escrita consistía en varios tipos de ítemes donde los estudiantes debían seleccionar el término más adecuado para diferentes contextos oracionales; escribir el significado de algunas palabras; anotar oraciones con distintos lexemas y realizar un pareo de significados entre varios términos.

\section{Actividades para ampliar el vocabulario}

Durante un lapso de cinco semanas, para el grupo experimental, y tres semanas, para el grupo control, se llevaron a cabo las siguientes actividades con el propósito de enriquecer el léxico de los estudiantes de octavo año.

1. Actividades introductorias y comunes a ambos grupos:

- $\quad$ En primer lugar se procedió a explicar a los grupos los contenidos léxicos que se establecen en el plan de estudios de español para el Tercer Ciclo de la Enseñanza General Básica.

- Como segundo punto se dio lectura a los cuentos El hijo y El difunto José, para observar el nivel de comprensión que los estudiantes tienen con respecto al vocabulario incluido en dichos textos literarios.

- Posteriormente, se les dictó a los estudiantes cuarenta de las palabras seleccionadas del corpus de los cuentos para revisar la ortografía de los términos.

- Se les planteó el proyecto de la elaboración de un diccionario con las palabras dictadas con anterioridad.

2. Actividades del grupo experimental:

Al grupo experimental se les aplicaron las siguientes prácticas para trabajar el vocabulario seleccionado con el fin de enriquecer su nivel léxico: Recuerde ¿Cómo se escribe y qué es?, ¿Qué estoy haciendo?, Lotería Léxica, La papa caliente y Recordemos vocabulario.

3. Aplicación de la pos-prueba:

Después de la realización de las actividades, ambos grupos realizaron las dos 
partes de la pos-prueba, las cuales son idénticas a las usadas en la pre-prueba.

4. Comprobación de la eficacia de las actividades:

Por último, se complementaron las actividades con la lectura de los cuentos elegidos, para observar si los estudiantes mejoraron la comprensión de los textos con el enriquecimiento de su vocabulario.

\section{Descripción de cada actividad}

A continuación se describen las prácticas de enriquecimiento léxico realizadas por el grupo experimental.

\section{Actividad 1: \\ Recuerde ¿cómo se escribe y qué es?}

\section{Descripción de la técnica:}

Se hacen subgrupos y a cada uno se le entrega una ficha con seis palabras del corpus elegido, que no conozcan y una hoja en blanco, dividida en seis partes, los estudiantes del grupo dibujarán lo que creen que significan esas palabras.

Una vez terminados los dibujos, los grupos se intercambian las hojas, con el fin de que el otro grupo identifique el término que se encuentra dibujado y lo escriba en la parte superior de cada dibujo. Todos los términos se encuentran escritos en un cartel colocado en la pizarra, para que los grupos determinen cuál de ellos se adecúa al dibujo hecho por sus compañeros.

Después, se devuelven las hojas a sus respectivos autores y ellos escriben el término que corresponda a cada dibujo.

Los términos que no fueron acertados por el grupo, se buscan en el diccionario y se escribe su definición, en la parte de atrás de la hoja.
Gana el grupo que haya dibujado con mayor acierto el término correspondiente.

\section{Actividad 2: Lotería léxica}

\section{Descripción de la técnica:}

La construcción del juego está a cargo del docente quien puede confeccionarla a mano o en computadora.

Se elaboran una serie de cartones con nueve cuadros, en cada uno de los cuadros se escribe una de las palabras que se extrajeron, con antelación de los cuentos vistos en clase. A su vez, se elaboran, en fichas aparte, las definiciones de cada uno de los términos.

En el aula, se entrega un cartón a cada estudiante o a cada grupo y con granos de frijoles o con un lápiz, ellos marcarán el concepto que concuerde con el significado que lee la profesora. Cuando algún alumno o grupo haya llenado el cartón, deberá gritar "Lotería". Si acertó todos los conceptos será el ganador.

Se puede jugar de diferentes formas para escoger el ganador, por ejemplo: a cartón lleno, a cuatro esquinas, diagonalmente, entre otras.

Una vez terminada la lotería, los estudiantes elegirán cinco palabras por grupo y harán un párrafo original y creativo. Se leen en voz alta y entre ellos escogen la mejor redacción.

\section{Actividad 3: ¿Qué estoy haciendo?}

\section{Descripción de la técnica:}

Se forman grupos y se solicita la ayuda de dos miembros de cada uno de los grupos, quienes deberán representar, por medio de mímica, el verbo que la profesora le indique. A cada grupo se le entregará una hoja en blanco, para que escriban el verbo que van adivinando. Gana el grupo 
que más verbos acierte y que no tengan errores ortográficos.

\section{Actividad 4: La papa caliente}

\section{Descripción de la técnica:}

Esta técnica es adecuada para repasar el léxico, pero también se puede usar para cualquier otro contenido visto en clase.

La profesora llevará tiras de papel en las que haya escritas oraciones incompletas, en las que se puedan utilizar las palabras que, con anterioridad, se han visto en clase. Las tiras de papel se meterán un una bolsa de plástico.

En un cartel, colocado en la pizarra, se encuentran escritas todas las palabras que completen las oraciones que están en la bolsa de plástico.

Los estudiantes se acomodarán en un círculo para ir pasando la bolsa de uno en uno. La profesora pondrá música y cuando la detenga, el estudiante que se quede con la bolsa sacará un papel, leerá la oración que está escrita y la completará con alguna de las palabras que se encuentran en el cartel de la pizarra. Si acierta la respuesta, el alumno ganará un punto. Si no acierta, introducirá, nuevamente la oración en la bolsa de plástico para que otro compañero tenga la oportunidad de contestar.

\section{Actividad 5: \\ Recordemos vocabulario}

\section{Descripción de la técnica:}

Anteriormente, en un trabajo extra clase, se les asigna a los estudiantes los términos desconocidos de los textos literarios que deban leer durante la investigación, para que el alumno busque los significados en el diccionario y confeccione su propio diccionario.

La técnica consiste en revisar la tarea de forma dinámica: el docente coloca en la pizarra algunas de las palabras y sus acepciones, en papeles de colores.

Se forman dos grupos para que en un lapso determinado coloquen el significado al lado de su respectivo término. Se elige un coordinador, que después de deliberar con el grupo, pone el significado al lado de la palabra correspondiente. Van por orden y gana el grupo que más significados relacione correctamente. Los coordinadores de los otros grupos señalan los posibles errores cometidos y se realizan las correcciones respectivas.

\section{Análisis de resultados}

\section{Tratamiento de los datos}

Para la presentación de las frecuencias de uso de las palabras seleccionadas de los cuentos se elaboraron cuadros comparativos que incluyen la cantidad de veces que los estudiantes utilizaron los términos, tanto en la pre-prueba como en la pos-prueba, para el grupo de control y para el grupo experimental.

Para este estudio, se tomaron en cuenta, únicamente, los usos adecuados que los estudiantes hicieron de cada una de las palabras escogidas y se aplicó, para el análisis cuantitativo, la prueba de "Comparación de proporciones de $\mathrm{t}$ de Student”, cuya fórmula es:

$$
\begin{gathered}
\mathrm{t}=\text { Ppre }- \text { Ppos } \\
\sqrt{\frac{\text { PpreQ }}{n} 1}+\frac{\text { Ppos Q }}{n 2}
\end{gathered}
$$

A continuación se presenta un resumen de estos hallazgos:

De acuerdo con la comparación entre los resultados obtenidos de la pre-prueba y la pos-prueba, se determinó que hubo lexemas cuya frecuencia de uso correcto no presentó variación alguna en el grupo experimental. No obstante, el resto de términos presentaron una variación leve o mayor con respecto a la utilización adecuada de estos. Las palabras que mayor diferencia 
presentaron, en cuanto a su frecuencia, se encuentran marcadas con un asterisco $\left({ }^{*}\right)$ para que puedan identificarse claramente.

\section{Cuadro 1}

Comparación de las frecuencias obtenidas en la pre-prueba y la pos-prueba del grupo experimental, con las cuarenta palabras tomadas para la realización de las actividades de enriquecimiento léxico

\begin{tabular}{|c|c|c|}
\hline \multirow[b]{2}{*}{ Conceptos } & \multicolumn{2}{|c|}{ Frecuencias } \\
\hline & Pre-Prueba & Pos-prueba \\
\hline 1. Candente & 12 & 12 \\
\hline 2. Detonación & 12 & $20 *$ \\
\hline 3. Quebrantar & 12 & 11 \\
\hline 4. Espartillo & 11 & $21^{*}$ \\
\hline 5. Posar & 11 & 11 \\
\hline 6. Sombrío & 11 & $22^{*}$ \\
\hline 7. Centella & 10 & $18^{*}$ \\
\hline 8. Porvenir & 10 & $12^{*}$ \\
\hline 9. Clamar & 9 & $14^{*}$ \\
\hline 10. Deparar & 8 & $9^{*}$ \\
\hline 11. Instancia & 8 & $15^{*}$ \\
\hline 12. Sienes & 8 & $20^{*}$ \\
\hline 13. Bastar & 7 & $11^{*}$ \\
\hline 14. Casal & 7 & $16^{*}$ \\
\hline 15. Ceñir & 6 & $8^{*}$ \\
\hline 16. Costear & 6 & $14^{*}$ \\
\hline 17. Deplorable & 6 & $11^{*}$ \\
\hline 18. Fusil & 6 & $17^{*}$ \\
\hline 19. Guápil & 6 & $17^{*}$ \\
\hline 20. Limar & 5 & $10^{*}$ \\
\hline 21. Rematar & 5 & $6^{*}$ \\
\hline 22. Tez & 5 & $15^{*}$ \\
\hline 23. Estampido & 4 & $14^{*}$ \\
\hline 24. Enrarecer & 3 & $7 *$ \\
\hline 25. Impregnar & 3 & $6^{*}$ \\
\hline 26. Recluir & 3 & $8^{*}$ \\
\hline 27. Abra & 1 & $7 *$ \\
\hline 28. Barranco & 1 & $7 *$ \\
\hline 29. Linde & 1 & $6^{*}$ \\
\hline 30. Radio de acción & 1 & $11^{*}$ \\
\hline 31. Agorera & 0 & $2^{*}$ \\
\hline 32. Amenguar & 0 & $6^{*}$ \\
\hline 33. Cinegética & 0 & $11^{*}$ \\
\hline 34. Esbozar & 0 & $1^{*}$ \\
\hline 35. Inexorable & 0 & $4^{*}$ \\
\hline 36. Nimio & 0 & $5^{*}$ \\
\hline 37. Percutir & 0 & $3^{*}$ \\
\hline 38. Proferir & 0 & 0 \\
\hline 39. Taciturno & 0 & $12 *$ \\
\hline 40. Vera & 0 & $14^{*}$ \\
\hline
\end{tabular}

Se tomaron en cuenta cuarenta términos de los cuentos "El hijo" y "El difunto José" para realizar las actividades de enriquecimiento léxico, con el grupo experimental. Una vez realizadas las prácticas con los 25 estudiantes del grupo y al aplicarles la pos-prueba, del total de cuarenta palabras, treinta y seis de ellas, es decir un $90 \%$ obtuvo un cambio significativo en la frecuencia de su uso, ya que de acuerdo con el análisis de las pruebas, se determinó que la mayoría de estas fue utilizada en mayor escala durante la pos-prueba que en la pre-prueba.

Gracias a este resultado se puede desechar la hipótesis cero Ho, que era establecer los resultados de la pre-prueba y la pos-prueba del grupo experimental como iguales y aceptar la hipótesis alternativa o $\mathrm{H} 1$, la cual establece las frecuencias obtenidas en la pos-prueba como mayores a las obtenidas en la pre-prueba para dicho grupo experimental, al 90\% de confianza.

En la primera parte de la preprueba, los estudiantes escribieron una redacción en que se incluían algunas palabras de las elegidas del corpus total extraído de los cuentos. Esta parte de producción textual es, para la profesora, mucho más interesante de analizar, pues permite comprobar la forma en que los estudiantes utilizan los lexemas, dependiendo del conocimiento del significado de las palabras o si lo hacen aún sin comprender con exactitud la definición de cada término.

Por ejemplo, en el grupo experimental, durante la aplicación de la pre-prueba, hubo alumnos que usaron los términos de la siguiente manera:

1. "Todo se nos enrarecía cuando llegamos..."

2. "Venían como una película en la que era sofocado por sus creencias..."

3. “..él divisó un fusil candente que lo seguía uno,..." 
4. "Era hora de que Inglaterra llegar a quebrantar las consecutivas victorias de Alemania."

5. “...un mal porvenir el cual me provocó que mi curiosidad se remataría y provocaría una detonación..."

6. "Entonces creí mejor mutilar el pasado..."

7. "Debemos ojear más detenidamente a los enfermos mentales..."

En los ejemplos anteriores se puede observar como los estudiantes, en algunas ocasiones, utilizan los términos sin pensar si la palabra concuerda con el contexto sobre el cual están escribiendo, de ahí que se sienta un uso forzado del lexema y no se compruebe si el alumno tiene un conocimiento exacto de su significado.

Por ejemplo, el verbo rematar está empleado incorrectamente, pues este fue utilizado para referirse a la curiosidad y, si se toma en cuenta su definición de diccionario, la curiosidad no puede rematarse, como sí puede rematarse una costura en la ropa.

El caso de mutilar, es un verbo que significa cercenar o cortar una parte del cuerpo ya sea humano o animal. Para mutilar se requiere de un objeto concreto que pueda ser destrozado, por tal motivo el pasado no puede ser mutilado porque es un elemento abstracto y además, inexistente en el momento presente.

Otro aspecto que se puede ejemplificar es el del verbo "ojear a los enfermos mentales". El hecho de ojear es realizar una acción de pasar rápidamente las hojas de un libro o ver superficialmente alguna situación; en este contexto se usa como si fuera una observación detenida, de ahí que su uso es erróneo.

Otra situación que se presenta con estos ejemplos es que los estudiantes tienden a poner varias palabras del corpus juntas, este es el caso de fusil candente, como forma de utilizar la mayor cantidad de palabras posible, dentro del contexto de escritura.

Una vez que se aplicaron las actividades, la perspectiva del significado de las palabras, que tenían los estudiantes, cambió de manera sustancial, pues el uso que les dieron a los conceptos, en la pos-prueba, se mantuvo en un nivel más elevado de desarrollo, como se puede observar en los siguientes ejemplos:

1. "También explican que él era un hombre taciturno."

2. "Tuvieron que quebrantar las leyes..."

3. “...esbozábamos una sonrisa..."

4. "Al emprender al día siguiente de nuevo mi camino, fui a costear toda la zona..."

5. “...un helicóptero costeó la isla..."

6. “...de dónde provenía la detonación..."

7. “...y al ver que era en vano buscarla, decidió continuar..."

En la pre-prueba, muchas de las palabras seleccionadas para la redacción, no fueron utilizadas, mientras que en la pos-prueba sí las incluyeron, pues su búsqueda en el diccionario y las actividades realizadas con los estudiantes, les ayudó a conocer su significado. Algunos de estos ejemplos son los casos de hombre taciturno y esbozar una sonrisa. Además, el verbo costear ya no sólo fue usado como acción de pagar por algo, sino de rodear o bordear un territorio determinado, como en costear la zona.

En el Cuadro 2 se representa la frecuencia con que los alumnos del grupo control usaron adecuadamente los cuarenta términos elegidos para la búsqueda en el diccionario.

De igual manera el cambio más significativo en las frecuencias se marcó con un asterisco (*) para su identificación. 


\section{Cuadro 2}

Comparación de las frecuencias obtenidas en la pre-prueba y la pos-prueba del grupo control, con las cuarenta palabras tomadas para la realización de las actividades de enriquecimiento léxico

Frecuencias

\begin{tabular}{|c|c|c|}
\hline Conceptos & Pre-prueba & Pos-prueba \\
\hline 1. Sendas & 13 & 11 \\
\hline 2. Detonación & 12 & 8 \\
\hline 3. Barranco & 11 & 4 \\
\hline 4. Casal & 11 & 11 \\
\hline 5. Deparar & 11 & 11 \\
\hline 6. Espartillo & 11 & $12^{*}$ \\
\hline 7. Fusil & 11 & $17 *$ \\
\hline 8. Instancia & 11 & 11 \\
\hline 9. Piapiá & 11 & $17^{*}$ \\
\hline 10. Posar & 9 & $12^{*}$ \\
\hline 11. Sombrío & 9 & $15^{*}$ \\
\hline 12. Clamar & 8 & $10^{*}$ \\
\hline 13. Limar & 8 & $11^{*}$ \\
\hline 14. Porvenir & 8 & $12^{*}$ \\
\hline 15. Centella & 7 & $14^{*}$ \\
\hline 16. Vano & 6 & $13^{*}$ \\
\hline 17. Cinegética & 5 & $6^{*}$ \\
\hline 18. Costear & 5 & $9 *$ \\
\hline 19. Reducir & 5 & $8^{*}$ \\
\hline 20. Ceñir & 4 & $5^{*}$ \\
\hline 21. Estampido & 4 & $11^{*}$ \\
\hline 22. Guápil & 4 & $16^{*}$ \\
\hline 23. Impregnar & 4 & 3 \\
\hline 24. Amenguar & 3 & 1 \\
\hline 25. Deplorable & 3 & $5^{*}$ \\
\hline 26. Enrarecer & 3 & 2 \\
\hline 27. Rematar & 3 & 3 \\
\hline 28. Abra & 2 & 1 \\
\hline 29. Inexorable & 2 & $3^{*}$ \\
\hline 30. Nimio & 2 & $7 *$ \\
\hline 31. Radio de acción & 2 & $11^{*}$ \\
\hline 32. Sienes & 2 & $12 *$ \\
\hline 33. Tez & 2 & $10 *$ \\
\hline 34. Percudir & 1 & 0 \\
\hline 35. Proferir & 1 & $2 *$ \\
\hline 36. Taciturno & 1 & $9 *$ \\
\hline 37. Agorera & 0 & $1^{*}$ \\
\hline 38. Esbozar & 0 & $1^{*}$ \\
\hline 39. Linde & 0 & $6^{*}$ \\
\hline 40. Vera & 0 & $6^{*}$ \\
\hline
\end{tabular}

Para el grupo control, conformado por un total de 27 alumnos, al igual que en el grupo experimental, se tomaron, para las actividades de enriquecimiento léxico, las cuarenta palabras que menor frecuencia de uso correcto lograron en la aplicación de la pre-prueba. De ese total de cuarenta palabras, veintiocho de estas, un $70 \%$, variaron su uso de forma importante (tomando en cuenta que la única actividad realizada con este grupo fue buscar el significado de los términos en el diccionario) de acuerdo con las frecuencias arrojadas con la aplicación de la pos-prueba.

De igual manera y con la utilización de la prueba de comparación de proporciones de $\mathrm{t}$ de Student, para el análisis cuantitativo, se descarta la hipótesis cero (Ho) y se acepta la H1, en donde los resultados obtenidos en la pos-prueba son mayores que los resultados producidos en la pre-prueba, al 90\% de confianza en dicha fórmula aplicada a las dos pruebas, es decir, el tratamiento aplicado al grupo experimental surtió un efecto positivo, en la frecuencia en que los estudiantes utilizaron las palabras seleccionadas para las pruebas.

Con respecto al grupo control, igualmente los educandos usaron muy superficialmente los términos en la pos-prueba, esto porque al grupo solamente se le solicitó que buscara las palabras en el diccionario y no tuvieron un mayor contacto con estas; contrario al grupo experimental, donde se practicó, en repetidas ocasiones, con contextos adecuados a cada uno de los términos seleccionados para el enriquecimiento léxico.

Los siguientes son algunos ejemplos tomados de las redacciones de la pre-prueba del grupo control.

1. “...y sus padres les costearon la boda..."

2. "Ella empezó a posar en el bosque de una manera de alucinación muy fuerte..."

3. “...ella empezó a clamar miedo.”

4. “...amigos ojeaban sus intereses y él no tenía derecho a ellos."

5. "Los del pueblo terminaron fusilados, mutilados y recluidos en celdas." 
6. "En el camino ojeamos a un señor posando y limándose las uñas."

7. "...corriendo a la escuela ya que no podía costear una bicicleta..."

Es importante observar el esfuerzo que los estudiantes del grupo control hicieron para poder utilizar la mayor cantidad de palabras, aunque, al igual que en el caso del grupo experimental, descontextualizaron un poco el significado de los términos o usaron otra acepción de estas que no se relacionaba directamente con el contexto en que aparecen en el cuento.

Ejemplos de esta situación son los casos de costear que remite a una definición económica; clamar no se refiere a hablar sino a sentir miedo y ojear, usado con el sentido de observar o vigilar por los intereses de una persona y no de ver superficialmente una situación o un hecho.

En la pos-prueba, en el grupo control, a pesar de no haber recibido las actividades de enriquecimiento léxico, los alumnos utilizaron satisfactoriamente los términos, en comparación con los resultados que arrojaron sus pre-pruebas, después de haber buscado las palabras en el diccionario; así lo comprueban los siguientes ejemplos extraídos de sus redacciones:

1. "Él entonces decidió ojear el bello paisaje..."

2. “...que remató un puñetazo en la cara del bravucón."

3. "Ambos deberían emprender un largo camino el cual debían costear el territorio..."

4. “...emprendía su largo camino."

5. “...prestaba mucha más atención y ojeaba sus revistas,..."

6. "...han clamando piedad pero nadie les responde..."

7. “...emprender su viaje al éxito y al llegar clamar con orgullo que sí se pudo.”

El uso que en el grupo control se les dieron a las palabras, en la pos-prueba, fue mucho más claro y contextualizado que en la prueba realizada con anterioridad, ya que los alumnos relacionaron satisfactoriamente el significado de los lexemas en un contexto de escritura.

\section{Conclusiones}

Realizar el proyecto de buscar los cuarenta términos elegidos del corpus en el diccionario fue una actividad muy útil en tanto, en el grupo control se logró elevar la frecuencia con que los estudiantes utilizaron adecuadamente dichos términos. Sin embargo, las prácticas que se aplicaron al grupo experimental tuvieron mayor éxito porque los alumnos lograron relacionar las palabras con sus significados y de esta manera, asociarlas a contextos oracionales determinados, con lo cual mejoraron su utilización y alcanzaron un alto nivel de comprensión de los lexemas, ya que las palabras usadas se vieron en un contexto y esto ayudó a entender mejor su utilización en situaciones comunicativas escritas y como parte de textos literarios.

Las actividades de Recuerde ¿cómo se escribe y qué es?, La papa caliente, Recordemos vocabulario y ¿Qué estoy hacien$d o$ ? fueron provechosas para lograr el enriquecimiento léxico de los estudiantes porque consistieron en prácticas entretenidas y de aprendizaje, en las cuales los alumnos estuvieron en contacto constante con oraciones en las que podían usar las palabras. Estas prácticas fueron muy dinámicas para ellos, de tal manera que se interesaron por participar activamente en su realización.

La Lotería léxica no surtió el mismo efecto que el resto de actividades porque fue una práctica memorística y los alumnos no se encontraban totalmente familiarizados con los conceptos, de manera que no pudieron identificar su significado y por ello no se produjeron los resultados esperados. Esta actividad solamente buscaba que los estudiantes asociaran las palabras con sus definiciones, por tanto fue mucho menos dinámica que el resto de prácticas. 
Fue importante realizar un número considerable de actividades con el corpus seleccionado, porque de esta manera los estudiantes estuvieron en un contacto continuo con las palabras y esto los benefició para lograr aprehender el léxico. No es recomendable utilizar menos actividades, sino aumentarlas y aplicarlas con un lapso que medie entre cada una, para no presionar a los alumnos a que estén utilizando siempre el mismo contenido, pero proponiéndoles diversas prácticas que enriquezcan su léxico con términos de todos los textos literarios que se ven en los diferentes cursos de la Enseñanza General Básica.

\section{Referencias bibliográficas}

Araya Calderón, Kattia. (2000). La enseñanza del léxico en los programas de Español del Tercer Ciclo del Sistema Educativo Costarricense. Tesis para optar por el título de Licenciatura en Filología Española. San José. Universidad de Costa Rica.

Ausubel, David y otros. (1983). Psicología Educativa. México, D. F. Editorial Trillas.

Coseriu, Eugenio. (1997). Principio de semántica estructural. España. Editorial Gredos, S. A.

Diccionario de la Lengua Española. Real Academia Española. (2001). Vigésima segunda edición. España. Editorial Espasa.
Geckeler, Horst. (1976). Semántica estructural y teoría del campo léxico. España. Editorial Gredos, S. A.

Novak, Joseph D. (1997). Teoría y práctica de la educación. España. Alianza Editorial, S. A.

Ortiga Ojeda, Gonzalo. (1996). "Implicaciones dialectológicas y didácticas del léxico disponible." En: $L a$ enseñanza del léxico español como lengua extranjera. España. Edición Iberoamericana.

Rojas Porras, Marta y otros. (1994). "Caracterización de los procesos de enseñanza-aprendizaje a partir del análisis de la interacción verbal en el aula costarricense.” En: Káñina. Vol. XVIII (2). Pág. 141-157. Rev. Artes y Letras. San José. Universidad de Costa Rica.

Sánchez Corrales, Víctor. (1993). "Campos semánticos y disponibilidad léxica en preescolares." En: Revista Educación. Vol. 17 (2). Pág. 15 - 25. Costa Rica. Editorial de la Universidad de Costa Rica.

Vences, Úrsula. (1996). “Al principio ya existía la palabra. Caminos autónomos e individuales hacia la elaboración y el aprendizaje del vocabulario." En: La enseñanza del léxico español como lengua extranjera. España. Edición Iberoamericana. 
